\title{
Entrepreneurship and Innovation: The Antecedents of Opportunity
}

\author{
Tina PHIRI and Brian BARNARD*
}

Wits Business School, University of the Witwatersrand (WITS), South Africa

\begin{abstract}
The study examines the antecedents of opportunity in greater detail. A literature review highlights 9 antecedents of opportunity, and it was further investigated how these factors impact opportunity recognition. Some of the leading antecedents of opportunity were: solving problems, goals and aspirations, artistic creativity, adoption and networking. None of the antecedents by themselves are sufficient for innovation, as each has a caveat that may also hinder innovation. Also, it is evident that a number of the antecedents may offer unique solutions and benefits, including the acceleration of the identification of ideas and opportunities. In certain contexts, one antecedent may be more applicable or beneficial than another. Each antecedent potentially offers and allows expert areas of innovation, with innovators becoming very experienced in a particular antecedent as an area of innovation. Consequently, the innovator may continually gravitate towards a certain antecedent. A number of themes common to the antecedents suggest that there may be a number of principal factors underlying the antecedents studied. Underlying all the antecedents may be the need to cross boundaries of the acceptable, or rather boundaries of the possible. Innovation may equally be defined as crossing the boundaries of what is possible, whilst still yielding outcomes that are desirable. The primary factors expected to underlie the antecedents are: 1) perspective, 2) knowledge, and 3) first person experience (sensing, interpreting). There may be a link between the expertise of the innovator, and antecedent use. Antecedents considered by innovators may shape and define the type and nature of innovation taking place in a sovereign.
\end{abstract}

Keywords: Entrepreneurship, Innovation, Antecedent, Opportunity, Opportunity Recognition, Problem Solving, Goals, Creativity, Adoption, Networking, Interests, Technology, First-Person Experiences, Synthesis

JEL Classification: O30, L26

\section{Introduction}

To date, the antecedents of opportunity have not been extensively researched. A literature review on innovation highlights a number of factors that contribute to the generation of ideas and opportunity recognition. The purpose of the study is twofold. Firstly, the research seeks to contribute to the current literature on the

\footnotetext{
${ }^{*}$ Corresponding Author:

Brian Barnard, Wits Business School, University of the Witwatersrand (WITS), South Africa

Article History:

Received 2 March 2018 | Accepted 23 May 2018 | Available Online 1 June 2018

Cite Reference:

Phiri, T. and Barnard, B., 2018. Entrepreneurship and Innovation: The Antecedents of Opportunity. Expert Journal of Business and Management, 6(1), pp.61-81.
} 
primary factors that contribute to opportunity recognition, and thus innovation. Secondly, the research sought to understand how these factors contribute to innovation - some of the positive aspects and the negative aspects of each antecedent.

\section{Literature Review}

Richards et al. (1988) note that creativity is identified by two widely employed criteria: 1) originality - new or unusual elements must be involved, and 2) adaptation to reality - outcomes must be meaningful to others rather than random or idiosyncratic. Creativity so defined may be identified in virtually any field of activity. Assessment need not be restricted to traditionally creative areas of endeavor (e.g., the arts and sciences) or to accomplishments that are socially recognized. They note two types of variables pertaining, respectively, to quality and quantity of creativity: measures of 1) peak creativity, and 2) extent of involvement in creative activity. Further, research on creativity can be grouped into four areas: the creative person, process, product, and press.

DiLiello and Houghton (2006) argue that individual creativity consists of two distinct elements: 1) creative potential; and 2) creative behavior. Creative potential refers to an individual's creative skills and abilities. Creative behavior or creative output, on the other hand, is the measured result of creative efforts. Creativity may be generally defined as the formation of novel, appropriate and useful ideas by individuals or small groups. A distinction exists between creativity in the context of truly novel ideas and creativity in the context of problem solving. Although both types of creativity are important to most organizations, creative problem solving is a more common type of creativity that is more accessible to most people and more widely applicable in organizational settings. Three components comprise individual creativity in a given domain: 1) domain-relevant skills or expertise (innate skills), 2) creativity-relevant processes (learned abilities), and 3) intrinsic task motivation (task attitudes). Creativity-relevant processes include: personality; cognitive style; work style; and the use of heuristics for exploring new cognitive pathways.

Kerr and Lloyd (2008) state that creativity is defined as the creative human attributes and qualities concerned with imagination, inventiveness, improvisation, insight, intuition, and curiosity - the natural artful genius and talent of people. Transformative learning is an adult learning process where, based on new knowledge and values, beliefs are critically examined, and the learner changes their frame of reference as they re-interpret their world. There are, at first look, two views of transformative learning, with one reflecting a rational approach of critical reflection, and the other relying more on imagination and emotion. Both use rational processes and incorporate imagination as a part of a creative process.

DiLiello and Houghton (2006) note that creative-thinking abilities include divergent-thinking, the use of association, analogies, metaphors and intuition. Creative problem solving techniques can be posited on a creativity continuum ranging from safe (paradigm preserving techniques such as brainstorming), to more imaginative and expressive paradigm-breaking techniques such as guided fantasy. McFadzean (1998a) notes that creativity techniques can be classified into two groups: analytical and intuitive. Analytically-oriented techniques are based on a structure to develop a logical pattern of thought. These techniques include ForceField Analysis and Progressive Abstraction. Intuitive techniques, on the other hand, allow the participant to make giant leaps or to observe images or symbols in order to arrive at a solution. Wishful Thinking and Metaphors are two techniques that can be classified under the intuitive category. Classical Brainstorming does not produce very many ideas that challenge or break away from a prevailing paradigm - this technique produces more paradigm preserving ideas than paradigm breaking ideas. This is because these techniques only use free association. They do not force the participants to use their imagination to develop ideas (although some may do so), but piggyback on the ideas of others, thus participants tend to follow a more structured process.

Below, 9 antecedents of opportunity are highlighted: 1) artistic creativity: creative inspiration, imagination, and objective fantasy, 2) interests, inquisitive journeys and passions (desire to understand something; being passionate about something), 3) solving problems (desire to solve a problem; desire to bring improvement or change), 4) goals, aspirations and values, 5) scientific and technical inquiry or development, 6) adoption (borrowing from other contexts), 7) synthesis and induction (cross-pollination of fields of knowledge; building up concepts), 8) first person experiences, and 9) negotiation and networking (partnerships, contacts, relationships).

\subsection{Artistic Creativity: Creative Inspiration, Imagination, and Objective Fantasy}

Chodorow (2006) argues that active imagination and creative imagination are basically the same process. Both involve the expression and transformation of the emotions. McFadzean (1998b) argues that humour can also help to stretch a participant's thinking and help to change his or her mind set. It can also force 
participants to combine ideas that were not associated together before. Baron (2007) notes that a well-known French film director once described the creative process in these words: "Make visible what, without you, might perhaps never have been seen." Ardichvili et al. (2003) argue that, where value sought is unidentified and value creation capability is undefined (problems and solutions are both unknown), it may represent the kind of creativity associated with artists, "dreamers," some designers, and inventors interested in moving proprietary knowledge in a new direction or pushing technology past its current limits. McFadzean (1998b) states that, when a problem is open-ended, some degree of fantasy can provide the degree of loosening needed to produce many unique ideas.

McFadzean (1998a) states that creativity can be enhanced by looking at the problem from a variety of perspectives and by breaking old mind patterns and forming new connections and perceptions. Breakthrough thinking also enables decision makers to view problems from a different perspective. It focuses on the future, that is the goals and purposes of the organisation or department, rather than what went wrong. This allows the decision-makers to view the problem from a different perspective and encourages the use of more creative problem solving techniques such as Excursion. Individuals examine colorful photographs or graphic words and try to link the images to the problem. Object Stimulation as technique involves the problem solving group developing a list of objects that are unrelated to the problem. The group describes each object and then uses these descriptions to stimulate ideas pertaining to the problem. The purpose of such stimuli is to present a completely different problem perspective. The presence of unrelated stimuli encourages participants to change their perspective of the problem. In Guided Fantasy, participants are asked to describe a scenario including their feelings, sounds, colours and smells. New ideas are developed from these scenarios and they are then linked back to the problem.

Yokochi and Okada (2005) essentially show that boredom can be a source of creativity and inspiration. Artists often become bored while producing similar works repeatedly, and tries something new for stimulation. They note how random lines created by an audience both constrains as well as motivates and inspires a certain artist. In studying craftsmen, Fillis (2007) notes a type of craftsman that can be described as an artist/ designer who is unwilling to view the craft as a product, but as a creative piece (the idealist). Although they may have initially created craft in response to market demand, they have spent time considering their philosophical position. Their stance tends to be uncompromising when producing the work; they do not tend to take note of customer demand, but instead make art/ craft which they feel has artistic integrity. In other words, they embrace an "Art for Art's sake" philosophy, rather than "Art for Business sake."

Tschang (2003) notes some of the skills and techniques game designers use: 1) problem setting or framing, 2) out of the box thinking, including techniques like imagining the reverse of a problem or objective, or bringing clashing ideas together, 3) visualization of dynamic consequences (of design decisions), 4) crosspollination, and 5) intuition. The thinking skills roughly fall into two categories: creative (e.g. intuitive or involving divergent thinking tools), and problem-solving or analytical (e.g. problem structuring or constraint satisfaction). Furthermore, in many instances, designers reported using both types of skills (analytical and creative) either together or in a back and forth way in the design process. This is apparently due to the nature of the artifact being technologically complex, yet requiring artistic or intuitive inputs. Furthermore, a designer may just design the fact that an explosion will take place somewhere in a play sequence when something triggers it, but the programmer could spend weeks just tweaking it to look just right to a player.

Yokochi and Okada (2005) note how an artist can judge his work by drawing in the air first, before drawing on the medium. The image is drawn in the mind first. Sketches are a good medium for reflective conversation with one's own ideas and imagery. Design is seen as a process in which each designer constructs his or her own reality by his or her own actions that are reflective, responsive, and opportunistic to the design situation. It seems that designers gradually form their images while drawing their sketches. From results across studies of famous artists and studies of architectural design, it may be possible to predict that artists, especially painters, do not have entire images of their works at the beginning, but they gradually form their mental image through drawing objects. People become constrained within the properties of existing examples in generating new ideas and concepts. In the case of artists, it seems that their previous artwork is a constraint toward creating a new style of work.

Kozbelt (2008) studies artists creating original drawings, and subsequently rates them according to creativity. Both groups started mainly by drawing objects from the array, but this was less frequent among artists in sessions leading to high-rated drawings. As sessions progressed, both groups drew objects from the array less; however, they showed different subsequent trajectories. Artists in the high-rated group worked more on imaginary elements, while artists in the low-rated group drew other visible elements like the table or cast shadows, consistent with the mainly still-life appearance of their final products.

Glaveanu et al. (2013) note that fundamental for the impulsion of writers is their need to express, to 
"tell" or "speak", to show the world and "stage" something. Also, the creative activity of artists generally starts from the impulsion to create or make, to "do" or "incarnate", and it is also fed by a curiosity to see and understand, to "find sensations", and a need to express. The whole process starts normally with a "vision" or idea. The "click" comes usually after a period of void, of wandering, and the exact trigger can take many forms (for example an image). This initial idea is nevertheless schematic, necessarily incomplete, and needs a time of reflection, documentation, and incubation. These initial stages of "conception" lead to the stages of "fabrication", starting with the first sketches (e.g., the maquette) and up to a "draft" and the final object. Ideas are typically tested and experimented with on the basis of drawings and material depictions of the work, in clay or cardboard. The end result is often a series, as the first piece does not "exhaust" the sought after sensation. Within these formal stages, there are numerous work procedures artists employ to realize their vision, including repetition, multiplication, permutation of elements and inversion, simplification, and change of medium. Most of the time, the process is based on repetitive gestures, which for some are a means of relinquishing intentionality and "subjective decision".

Glaveanu et al. (2013) further note that the work of designers has its roots in similar impulsions to "make" things, to "build", and also to "touch," - to work with one's hands. The need to create and to be original and generate a "surprise" was also noted, as well as the need to transform, change, and experiment. Just as in the case of artists, the designer's work starts from an idea, an "intuition," the "embryo" of the final form. First externalizations of ideas take the form of drawings and sketches, and are progressively followed by 3D computer modelling and/ or physical mock-up. On the basis of these steps, the form is perfected and several of its details are progressively defined. The repertoire of designer work procedures involved in the stages above is extensive: amplification, deformation, association of ideas, use of allegories, schematisation, and simplification, the re-use of shapes, synthetic thinking and plenty of calculations.

Glaveanu et al. (2013) state that composers are left to establish their own "sound palette," looking for "sound actors" that will later be placed in a corresponding "script". Writing them down, the composer is then waiting for an "idea", for a "click". This is the second stage, when insights emerge out of an "accumulation of things" and one idea attracts another. More often than not, musicians work with ideas in plural, rather than one single "vision", as compositions have a time dimension. Given that there are many ideas or themes to work with, a moment of organization and reflection becomes necessary: distancing yourself and trying to make a plan.

Horng and $\mathrm{Hu}(2008)$ note that some of the culinary artists they studied, said that once they had several ideas to work (or play) with, they would begin with a vague concept or feeling and move toward clarity through the incubation of the idea. This may be the sort of process that cognitive psychologists would analyse in terms of retrieval and associative mechanisms, mental synthesis and conceptual combination, mental transformation and analogical transfer. During this phase, imagination might be more important than knowledge: Using one's imagination can lead to strategic solutions, which involve dynamic, creative action-based decision-making processes that introduce new modes of thinking.

Sawyer (2000) notes Picasso's improvisational style, called problem-finding by creativity researchers, is used by most successful painters. A "problem-finding" painter is constantly searching for his visual problem while painting - improvising a painting rather than executing one. In contrast, a problem-solving style involves starting with a relatively detailed plan for a composition and then simply painting it; "problem-solving" because the painter defines a visual problem for himself before starting, with the execution of the painting consisting of "solving" the problem. Improvisational interaction is highly contingent from moment to moment. An improvisational theatre performance is also, of necessity, a problem-finding process - albeit a collective one, akin to a brainstorming session. Similarly, a craftsman is problem-solving, whereas an artist is problemfinding.

\subsection{Interests, Inquisitive Journeys and Passions - Desire to Understand Something; Being Passionate about Something}

Grant and Berry (2011) state that intrinsically motivated employees use interest as a guide for determining which ideas to pursue, and that interest primarily directs attention to ideas that are novel. Interest attracts people to new, unfamiliar things, and many of these things will turn out to be trivial. Hirst et al. (2009) argue that a learning orientation focuses individuals on the acquisition of new knowledge and the development of "deep-processing strategies" that facilitate mastery of challenging tasks. The focus on skill development associated with a learning orientation implies an intrinsic interest in understanding and mastering task performance. This interest in the task itself - intrinsic motivation - leads to a deeper and more intensive engagement with the task, which often results in creativity. Because a learning orientation is also associated with a preference for challenging and demanding tasks, individuals with a stronger learning orientation may 
be expected to be more intrinsically motivated to seek out creative activities, that by definition involve uncertain and untried approaches that possess a high likelihood of error or potential failure. Baron (2007) argues that passion - entrepreneurs' powerful commitment to their new ventures, and a factor that might predispose them to experience rapid and intense changes in affect - plays an important role in the success of these companies. It can thus be argued that passion also positvely affects creativity.

In studying Celtic craftsmen, Fillis (2007) notes that for some, the business has evolved from a part time interest or pastime. In one particular case, it was just a hobby which developed. Glaveanu et al. (2013) note that many scientists also mention their passion for the domain of their choice and the pleasure they derive from working within it, their need to go further in their domain, to arrive at new and different results.

Tschang (2003) notes that some designers do research to back up an initial vision or idea, while in rare cases, like Will Wright's, research is done in order to uncover the new concept itself (as it emerges in the designer's mind). Research is sometimes linked to interests. For instance, Will Wright's years-long research on systems dynamics and other forms led to his path-defining SimCity, while his research into new architectural paradigms led to his recent The Sims, the biggest PC game seller in history.

Horng and $\mathrm{Hu}(2008)$ note an interview with Paul Liebrandt, an award-winning chef in New York. This chef's passion for cooking came from the busy, yet also free and affectionate atmosphere in the kitchen. This was, after all, an atmosphere conducive to sharing ideas with others and acquiring new ideas as well as new techniques virtually every day. Liebrandt's creativity seemed to come from his continuous learning, his willingness to listen to all the ideas and then to select from them those which he could use; in this way he was able to fashion his own style, but only because he had a solid foundation in culinary knowledge and praxis.

McFadzean (1998b) suggests that, to increase creativity, people should be allowed to spend time on their pet projects so they can be researched and developed. It is noted that the Post-It Note was developed by $3 \mathrm{M}$ because the company had allowed its inventor - Arthur Fry - to spend time working on the concept. 3M allows 15 percent of time to be spent on researching pet projects. In a study of Google, Vise (2007) notes that, right from the founding of the company, Brin and Page had decreed that all software engineers employed by the company should spend at least 20-percent of their time - one day a week - working on whatever projects were of interest to them. Brin and Page thought this would send the right signal that Google was a place where bright people could come and do some interesting stuff. The inspiration for this rule came from the fact both of the co-founders had been raised in homes where their fathers were on university faculties where they spent four days a week lecturing and one day each week on their own research. The 20 percent time was invented for people to just explore. People are productive when they are working on things they see as important or they have invented, or are working on something they are passionate about.

\subsection{Solving Problems (Desire to Solve a Problem; Desire to Bring Improvement or Change)}

Rank and Frese (2008) summarize the literature on the effect of negative affect on creativity. It is argued that feelings of tension and dissatisfaction may be needed for creative problem-solving. It is also noted that negative affect may function as a motivating force for creativity indicating that tension needs to be reduced, as a cue that a problem exists that needs to be solved or as content to be worked with in artistic productions. Distress-related variables at the individual, group and organizational levels of analysis act as a trigger for innovation. Innovative coping implies an individual perceives himself intentionally to introduce and apply, alone or within a group, new skills or procedures, designed to significantly benefit self, the group or the organization, with the result that recognized external demands appraised as taxing or exceeding resources, are actually reduced or alleviated. The groups of stressors that elicited innovative coping most frequently are overwork, procedural difficulties (e.g., problems with administrative tasks) and interpersonal problems in dealing with others. Stressors may be viewed as options for innovation and initiative, when they serve as signals that a process is not optimal and can be improved.

Glaveanu et al. (2013) argue that designers are motivated by a desire to solve a practical problem, and through this to respond to a certain need, coming from a client. Also, the impulsion that drives scientists toward their work mainly concerns the need to "solve," to find an answer to a problem or question and learn something new, coupled with great curiosity. Grant and Berry (2011) argue that, when employees focus their attention on others, they will be more likely to develop ideas that are ultimately useful to others. Prosocial motivation is an other-focused psychological process that directs employees' attention toward others' perspectives on what is useful, enhancing the impact of intrinsic motivation on creativity. Prosocial motivation is the desire to expend effort based on a concern for helping or contributing to other people.

Ardichvili et al. (2003) state that, when value sought is identified, but capability is undefined (problems are known but solutions are not), it describes situations in which structured problem solving, including information search, occur. The goal of opportunity development in this situation is usually design of 
a specific product/ service to address an expressed market need. Ward (2004) notes that other creativity models include steps such as problem construction, problem definition, and problem discovery. Implicit or explicit in these models is the belief that the way in which people conceptualize a problem strongly influences their likelihood of achieving an original or creative solution. Sutton (2002) notes many successful ideas were invented because someone got upset about something and then did something about it.

In depicting the story of Google, Vise (2007) notes that, since both Page and Brin were the sons of professors, they had always just naturally assumed they too would stay in academia for the balance of their careers. Brin's advisor, Professor Motwani, suggested a good subject for a doctoral thesis would be to look at finding new ways to extract meaningful information from large mountains of data. They were trying to solve interesting problems and stumbled upon some great ideas. Larry added ideas, Sergey added ideas, their PhD adviser added ideas, and it became clear they could build a full-scale search engine.

Hargadon and Bechky (2006) recall that, in 1988, Reebok hired Design Continuum to develop a successful response to the introduction of the Nike Air line of basketball shoes. Rather than develop a product design that was similar to Nike's concept, Design Continuum created the Pump shoe, a form-fitting shoe that worked because of an inflatable air bladder built into its sides. This idea first emerged in a brainstorming meeting when one of the designers, who had previously designed an inflatable splint, suggested that by building ankle support into a basketball shoe such splints might prevent injuries.

Sternberg et al. (2003) argue that, redirecting the concept of advertising children's toys saved Mattel from going out of business in the 1930s. The toy company - which was years away from making Barbie Dolls - was close to bankruptcy but made the wise decision to invest nearly all of its assets in the sponsorship of television's The Mickey Mouse Club. What Mattel decided was to take existing advertising strategies and redirect them. In the past, toy companies had tried hardest to sell their products to wholesalers and store buyers; if the toy store buyers did not choose to stock a particular toy in the store, then it was not sold. If the toy companies were going to target any public audience, it would be parents. However, by sponsoring The Mickey Mouse Club, Mattel would reach children directly. Mattel advertised its new Burp Gun on the show. The reaction was instantaneous. Children all over America demanded (and got) the toy. Within a few weeks, Mattel could barely keep up. In a few months, Mattel had sold more than a million Burp Guns. Mattel successfully redirected the field of children's advertising, moving the emphasis from reaching store buyers and parents to reaching the children directly.

Sternberg et al. (2003) also note that Henry Ford first launched his constant motion assembly line for the manufacture of the Model T Ford in 1913. Whereas large machines had previously been mass produced from a single location to which large stocks of components were hauled, Ford's innovation was to install a moving assembly line in which identical parts could be added to the car as it passed down the line. On the assembly line, one of Ford's Model T's could be produced in 93 minutes. This reduction in time led to a commensurate reduction in cost to the consumer. In particular, this unprecedented cost effectiveness forced Ford's competitors to join him and play the new production game. While his application of this technique to the automobile industry is no great feat of creative thinking, its impact on the field was strongly redirective. Ford's promotion of the assembly line and of mass production techniques was an impetus with wide-reaching impact. Whereas others had begun using these methods, Ford's introduction of the assembly line and mass production to the production of cars initiated a change in manufacturing whose impact reached beyond the automobile industry to include a broad range of industrial domains.

\subsection{Goals, Aspirations and Values}

McFadzean (1998b) states that creative thinkers look into the future and visualise where they would like to be in five or ten years time. McFadzean (1998b) further argues that the climate and culture within the organisation must be such that the company encourages innovation and creative thinking, and notes that one of the ways to do this is to establish a climate of excellence. This equally proposes that a climate or culture of excellence should lead to and encourage innovation and creativity. The propulsion model of Sternberg et al. (2001) lists eight types of creative contributions that can be interpreted as visionary: replication, redefinition, forward incrementation, advance forward incrementation, redirection, reconstruction/ redirection, reinitiation, integration.

McFadzean (1998b) notes that Wishful thinking as creative problem solving technique forces participants to look at a "perfect future". By using this method it allows group members to develop a goal that can be attained. The group members are asked to write a brief statement of the problem. The facilitator tells the group to assume that everything is possible. Each individual then needs to develop some fantasy statements about the future using terms such as: In the future, it would be nice if the organisation did... What really needs to happen to be a great company is... If I were in charge of this situation I would do... The group members 
must then examine each fantasy statement and develop ideas on how these can be achieved.

McFadzean (1998b) also describes Rich pictures as another creative problem solving technique. The group members are asked to write a brief statement of the problem. The facilitator then asks each individual to draw two pictures. The pictures may be a metaphor of the situation, e.g. a vehicle or an animal. The first drawing would be a picture of how each participant would like to see the situation in the future. The second picture would be a drawing of how the participants see the present situation. Each participant is asked to describe the picture of the present first. Not only should he or she describe the picture but a description should also be given of the properties of the objects drawn and why they have been drawn that way. Next, a description of the picture of the future should be given. Again, the properties and the relationships of the objects should be described. From the descriptions given by the participants new ideas can then be generated.

Tschang (2003) notes that some of the leading game designers that they interviewed, discussed at length what they sought to make in their games. As their career progressed, they appeared to be reflecting more on what makes their (or other) games fun or to be able to stand out. Some of these "player models" or game design philosophy included: realizing a more complete and consistent fantasy world (Tilted Mill), attempting to make music accessible to non-musicians (Harmonix), creating multiple optional paths in a game (Irrational), creating emergence (unintended dynamics) (Gas Powered Games), modeling thinking and interactivity (Chris Crawford), and building experiences based on alternate recreations of life (Carbon 6). As can be seen, these goals seem almost like quests that lead the designers to evolve their art and technology to greater forms.

Vise (2007) notes that Sergey Brin and Larry Page, the two young co-founders of Google never actually set out to change the world. When the company was founded in 1998, it didn't even have a business plan or a definitive business strategy. Instead, all Brin and Page had was an intense desire to do something innovative and to create a workplace where the best and brightest people could do some fun stuff. Vise (2007) recalls the co-founders: "Google is not a conventional company. We do not intend to become one. We aspire to make Google an institution that makes the world a better place. We are in the process of establishing the Google foundation and intend to contribute significant resources to the foundation, including employee time and approximately 1 percent of Google's equity and profits. We hope someday this institution may eclipse Google itself in terms of overall world impact."

Lumpkin and Lichtenstein (2005) note that, based on his research of the fledgling industry in 1951, the founder of the Vanguard Group, a leading-edge mutual fund management firm, concluded that a low-cost strategy could pay off in the long term. This cost-cutting approach was a driving force for a variety of experiments John Bogle enacted in his tenure as CEO of the organization. The program paid off during the stock market crash of 1987, during which virtually all Vanguard clients were served without a major glitch.

Lumpkin and Lichtenstein (2005) also recall The Natural Step, an entrepreneurial organization that has significantly advanced the movement toward environmental sustainability in Sweden, and, more recently, in the United States. The organization's CEO wanted to develop scientific guidelines for sustainability that could be understood by non-scientists and applied in business. However, given the prevailing industrial-age assumption that environmental and economic gains are mutually exclusive, he recognized that conventional decision-making approaches would be inappropriate. Through a highly iterative process of collaborative dialogue, a consensus document emerged that was endorsed by 50 of the top scientists in Sweden, and, at the same time, was clearly understandable to public figures in education, politics, and business. Soon a network of business leaders and others, encouraged by the king of Sweden, provided funding to disseminate the colourful booklet and audiotape to the entire population of Sweden. In addition, several of the supporting businesses including IKEA, Scandic Hotels, and Electrolux have led the country in developing highly innovative products that are ecologically sustainable and commercially successful.

\subsection{Scientific or Technical Inquiry or Development}

Glaveanu et al. (2013) state that, as a precondition in science, all respondents experienced long years of formal education, of "apprenticeships" that helped them build their "general scientific culture". The undergoing before doing is completed by a more informal and continuous process of learning beyond one's academic training. Reading books and articles, going to seminars and conferences are fundamental. "You are always forced to learn something" and get to monitor and "devour" technical progress. There is "enormous library work" before a study, because you do not want to get to demonstrate what has already been demonstrated. Discussions with colleagues supplement this effort and help ideas emerge or take shape. At times, certain concerns and questions are "in the air" within the scientific community, and scientists pick them up and work on them. Contrary to the popular image of the lone genius, if there is anything that defines scientific work, it is the fact that it always happens with others, alongside others, in relation to others. First points of contact are colleagues and peers, who play a crucial role in proposing problems, formulating ideas, 
clarifying them, orienting the work and finally evaluating it. "There is a collective dimension and teamwork in the process of creation" and often it is the case that two or more people work together and have an ongoing critical exchange.

\subsection{Adoption (Borrowing from other Contexts)}

Ardichvili et al. (2003) argue that, when value sought is unidentified, but capability is defined (problems are unknown, but solutions are available) - usually identified as "technology transfer" challenges, that is, capabilities and idle capacity in search of an application - opportunity development emphasizes search for applications more than product/ service development.

Gentner (2010) states that, according to structure-mapping theory, human comparison involves a process of establishing a structural alignment between two represented situations and then projecting inferences. The commonalities and differences between two situations are found by determining the maximal structurally consistent alignment between the representations of the two situations. As a natural outcome of the alignment process, candidate inferences are projected from the base to the target. These inferences are propositions connected to the common system in one analog, but not yet present in the other. Achieving a structural alignment sets the stage for four kinds of learning: abstraction, contrast, inference-projection, and re-representation. In abstraction, the common system resulting from the alignment becomes more salient and more available for future use. In contrast, alignable differences - differences that occupy the same role in the two systems - are highlighted. Inference-projection occurs when one member of the pair is more complete in its structure than the other; in this case, spontaneous candidate inferences will be made that enrich the lesscomplete item. A further way that learning can occur is re-representation: If there is good reason to believe two (non-identical) relations should match (e.g., a very good overall structural match), then one or both of the nonmatching predicates may be re-represented to permit the overall match.

Hargadon and Bechky (2006) argue that individuals facing problematic situations find solutions through a process of analogical reasoning, of trying to make sense of a new situation (the target analog) by relating it to a more familiar one (the source analog). Analogical problem solving occurs when an individual recognizes similarities in the new situation to old problems (and their solutions) that he or she has learned in the past. Transferring these existing solutions from old problems provides a solution to the new problem. Ward (2004) argues that analogy, or the mapping of knowledge from a familiar domain to a less familiar one, is central to creative developments in science, art, music, and literature and may also have applicability to entrepreneurship, as when a new successful venture is based on the principles that operate in other current successful ventures.

Glaveanu et al. (2013) note scientists have two broad options in their work: to develop a new technique or to use what already exists. As for the second option, this can be done either by applying existing theories and models to recently discovered phenomena or by modifying or adapting procedures to fit the problem at hand. More specifically, scientists strive toward parsimony, breaking down more complicated problems into simple ones and work, by making connections between problems and domains.

McFadzean (1998b) notes object stimulation and metaphors as paradigm stretching techniques and creative problem solving techniques. Object stimulation is an idea generation technique that can be used to explore the problem space as well as to enhance solution development. The technique encourages participants to view the situation from a different perspective by using unrelated stimuli. The group members are asked to develop a list of objects that are completely unrelated to the problem. Each individual then needs to select one object and describe it in detail. The group should use each description as a stimulus to generate new and novel ideas. Metaphors can be used to create a fantasy situation so that a new perspective of the problem can be gained. The group members are asked to write a brief statement of the problem. The facilitator asks the group to select a metaphor category or he can stipulate the category to the group (e.g. using the journey metaphor). Each individual then needs to describe the situation using the metaphor category. The facilitator needs to stipulate whether the description should be of the present situation or the ideal situation. Using the descriptions developed by each team member, the participants can generate new ideas.

McFadzean (1998b) also notes that creativity can be boosted by upsetting the status que, for example, by employing people who do not seem to fit in. It encourages people to look at situations from different perspectives instead of a "corporate viewpoint". This may not necessarily be comfortable for management but it can help the company produce some excellent innovative ideas. Hargadon and Bechky (2006) state that organizations that span multiple industries are able to generate creative ideas by gaining access to ideas in one domain and applying them in others. These firms' innovative accomplishments result from their work practices that transfer ideas over time and across projects. They note that the comments of someone from across the organization changed the way a project team at McKinsey working with a medical products company had been 
framing the problem of inventory management. A partner described how her reaction, after listening to the problem, was "Gee, by the way, this problem you're facing is the same one we faced when working with frozen foods." Within such interactions, introducing an alternative frame - and reflecting upon it - makes new aspects of the situation salient to other participants, prompting them to view the relevance of their past experiences in a new light.

Ward (2004) notes that commonly cited examples of analogy in creative endeavours abound, such as Rutherford's use of a solar system as a model for how the hydrogen atom was structured and Robbins, Laurents, Bernstein, and Sondheim's adaptation of Shakespeare's Romeo and Juliet to the context of a 1950s New York City gang conflict in West Side Story. Meticulous case studies have also detailed the role of analogy in major creative accomplishments, such as Kepler's reasoning about planetary motion, Edison's development of an electric light distribution system, and the Wright brother's efforts to craft a workable flying machine. Not surprisingly, then, analogy has been a key ingredient in proposals for enhancing creativity and has been listed as a component process in cognitive process models of creativity.

Holyoak and Thagard (1997) describe thinking by analogy: trying to reason and learn about a new situation (the target analog) by relating it to a more familiar situation (the source analog) that can be viewed as structurally parallel. It is also shown how analogical reasoning can be used to predict or describe possible outcomes, scenarios, or characteristics. The use of hopeful metaphors for clients' problems suggests that people can map themselves to persons or situations in ways that suggest solutions to personal problems. Empathic therapy may also involve analog retrieval or construction, when therapists have to work hard to find situations in their own lives that are semantically and structurally similar to that of the clients. The use of empathy in therapy involves an analogy between the client and the therapist, but the therapist may also find useful analogies between the client and someone else, perhaps a previous client with a similar problem. Such a mapping may help the therapist better understand the current client, and may also be used to provide a role model that the client can use as a source analog to suggest steps toward recovery.

Horng and $\mathrm{Hu}(2008)$ state that objects or elements can also be reduced (via categorical reduction) to more primitive categorical descriptions. A creative culinary artist can, for example, use the form of Chinese chess in the design of his or her bread or chocolate sculptures. The culinary artist, in creating a new dish, will naturally (spontaneously) search for precreation structures from which he or she can create unique designs. Creative culinary artists liked to think about an experimental structure in new or different contexts as a way of gaining insight into other possible uses or meanings of the structure.

Tschang (2003) argues that analogical thinking in the sense that is conventionally studied (e.g. a scientist mapping past knowledge onto a new situation) is only partly seen in game design. In game design, it is trickier to identify analogies as they do not just occur within a clearly demarcated context such as science. Sometimes, a designer directly uses a metaphor or real world analogy in a situation, or remembers a past incident that might help this particular design problem. However, other forms of analogy are more likely to take place, as when a designer directly adopts a feature seen in other games into his or her game (but possibly even in a different genre). At least one designer (RockStar Games) noted that he reused ideas wherever possible, and yet the designs he participated in were sometimes considered substantially innovative. Another designer for Tilted Mill noted that they tried to bring as much of the fantasy environment and experience (from either books like Tolkien's or "pencil and paper games") into their games. This latter is a form of analogical thought, but rather than translating from one situation to another, they are actually translating from a different medium, and in the process, also transforming the concepts. This type of analogical thinking is quite common as games are essentially constructed from (the designers' experience) of different media. A typical game involves some combination of narrative (i.e., story), character generation, sound, visual effects, and gameplay. Each of these presents an opportunity to form analogical material in games from their original media (i.e., books, books and movies, movies, music, and other games respectively). From the interviews, designers were constantly relying on their past backgrounds and experiences (e.g. material from side interests in reading) for their design work.

Tschang (2003) further notes that imitative games also require a type of analogical thinking involving the direct translation from one context (i.e. a previous game's design) to a new context.

Lumpkin and Lichtenstein (2005) detail the origins of Starbucks Corporation, which originated through a reconceptualization of the U.S. coffee industry by founder Howard Schultz. Based on his 1983 trip to Milan, Schultz recognized "an enormous opportunity for Starbucks to recreate the Italian coffee bar culture in the U.S.". The opportunity was to reframe coffee drinking into a social experience in America by providing a high-end product in a personalized environment to consumers wanting "affordable luxury". In this way, Schultz redefined the coffee industry in America, which, for more than the past 40 years, had been led by a few large companies competing on price and delivering a low-quality commodity that was meant to be made 
and consumed at home. By importing into his company the knowledge he gained from his (external) sources in Italy, Schultz developed a new framework for entrepreneurial action that was composed of several strategic assets gained through cognitive learning.

\subsection{Synthesis and Induction (Cross-Pollination of Fields of Knowledge; Building up Concepts)}

Ardichvili et al. (2003) argue that when value sought is identified and capability is defined (both problems and solutions are known), opportunity development involves matching known resources and needs to form businesses that can create and deliver value. Baron (2007) argues that concepts can be combined, with the result that something both new and useful is produced. For instance when the concepts of "telephone" and "camera" were combined, the idea for cell phones with built-in cameras emerged.

Ward (2004) states that work on conceptual combination reveals that when two previously separate concepts or images are merged into a single unit, novel properties can emerge that were not obviously present in either of the separate components, and that the effect is particularly strong for dissimilar or divergent concepts. Such novelty can be exploited to develop new product ideas or market niches. A combination does not have to involve verbal units at all to be a stimulus for creativity. Merging visually presented abstract forms, for example, can also lead to emergent new ideas. Fillis and Rentschler (2010) note that this closely relates to the notion of transmutation of thought and bisociation, while analogy has connections with the use of metaphor in rationalising uncertainty. As with metaphorical construction, analogy works best when there is a deeper level connection between the domains, rather than merely at the surface level.

McFadzean (1998a) argues that one method of encouraging creativity is to bring together teams of people so that participants can spark off new ideas from other group members as suggestions are made. Top quality ideas have also been shown to occur when groups use a group support system that allows the participants to remain anonymous. Creativity is enhanced when experience, ideas and diverse elements are mixed together and then transformed using new combinations. This is known as association and is the basis of many creative problem solving techniques.

George (2007) notes how unconscious thinking contributes to creativity. Imagine a developer is trying to come up with an initial prototype for a new concept and line of dishwashers. While consciously working on this task, the developer identifies a number of features of form and function and options for the line that seem viable, but not terribly exciting or unique. Not under a pressing deadline, the developer decides to put the project aside for a while and complete other tasks with deadlines. While putting together an unrelated PowerPoint presentation a few days later, an idea for the prototype that combines elements of the previously identified options in a unique manner pops into his mind. In each of these cases and countless examples from personal experience, conscious and unconscious thought work together.

DeTienne and Chandler (2004) note that the association perspective suggests that "applying procedures from one area of knowledge to another can give rise to novel associations and these associations can form the basis of creative ideas". An example of this might be the well-known method popularized by Edison in his expectations of his new assistants. Edison would ask new assistants to spend their morning wandering through the village writing down 20 things that interested them. After returning to the lab they would be asked to list 10 items in each column and then combine the items, two at a time, and seek to "discover" an invention which combined those two ideas. Baron (2007) states that concepts can be expanded. For instance, the concept "service station" or "gas station" used to mean just that: a place where fuel could be purchased and perhaps routine service performed on automobiles and trucks. Now, however, it has been expanded into places in which a wide array of other products, from food to clothing, can be obtained. Concepts can also be expanded by analogy - by perceiving similarities between objects or events that are otherwise dissimilar. For instance, when one scientist observed how plant seeds and burrs clung to his clothing with tiny hooks, he reasoned that this system would also work in other contexts, and Velcro was born.

Tschang (2003) notes that the piecemeal approach to game design may involve analogical thinking as well as additional skills like synthesizing abilities, that is, to be able to weave concepts from different media and past games into a coherent and "fun" whole. Even imitative games and fairly innovative games can be seen to be developed in a constructivist way, that is, by piecing together elements from previous games, designer's experiences, and work. The piecing together of a game comes about not so much through a brilliant insightful creative episode, than through a mixture of high concept, game design, and creative problem solving.

Horng and $\mathrm{Hu}(2008)$ state that culinary artists can combine single concepts to form more complex ones, and the meanings of one or more of the initial concepts can be altered as a result. The components of creative culinary works can be mentally rearranged and reassembled, and forms can be rotated or otherwise altered in shape to make interesting and potentially useful structures. This stage also involves the closelyrelated processes of analogical transfer and categorical reduction: A relationship or set of relationships in one 
context gets transferred to another, and thereby a new structure is created.

Sutton and Hargadon (1996) note that Brainstorms help IDEO designers acquire, store, retrieve, adapt, and combine knowledge of potential solutions to design products. As consultants to over 40 different industries, IDEO does detailed design work in many industries and does not specialize in one. They see a wider range of technologies than designers who develop products for one industry or organization. In network theory language, they are "well connected in several networks, rather than extremely central in just one". As a result, much of their value to clients is as a broker, or a bridge, transferring information between previously unconnected sources of technical knowledge. IDEO acts as a "technology broker" by moving technical knowledge between industries. Yet, technology brokering at IDEO means more than just acquiring and transferring knowledge between diverse industries. Engineers store this knowledge, retrieve it from storage, blend it, and adapt it for new design problems. IDEO's network position facilitates such creativity because designers have a storehouse of tried-and-true solutions from many industries that they can draw on to use in new ways.

\subsection{First Person Experiences}

Baron (2007) argues that pattern recognition appears to be closely related to opportunity recognition in the domain of entrepreneurship. This process involves noticing meaningful patterns in complex events, trends, or changes. In essence, it includes 1) recognizing links between trends, changes, and events that appear, at first glance, to be unconnected, and 2) noticing that these connections form an identifiable pattern. Affect may also play an important role in new venture creation. George (2007) argues that moods provide people with information. Positive moods provide information or signal that all is well, good progress has been made, and current efforts are sufficient. Alternatively, negative moods provide information that current efforts might be insufficient and thus, lead to lower levels of confidence in the progress that has been made on creative-idea generation. Thus, using their mood as input, people in negative moods might exert high levels of effort to come up with truly creative ideas.

Moran et al. (2003) note that, through play, children learn to give meaning to objects, to tease out relationships, to try on and practice different roles, to exercise their growing capabilities. In short, "play is the best preparation for future life - play is self-education". Tschang (2003) notes that, in the game development industry, being a game player is essential to making good, appealing products that resonate with players. Inspiration can come through an act of stimulation. Sutton and Hargadon (1996) note that they watched two videotaped brainstorming sessions on designing skin-staplers for surgery. The facilitator showed a videotape demonstrating the client's current stapler. He passed around the client's stapler and five or six competing staplers, while he explained the product and industry. Designers played with the staplers throughout the meeting and asked many questions. Another facilitator asked participants to create a "new fishing experience" that was easier and more fun for novices. The facilitator first got ten people on the roof with fishing rods in their hands, practicing casting and experiencing what it was like.

Lumpkin and Lichtenstein (2005) recall the story of Philadelphia Pharmacy: One day, its founder, Leon Ost, found an assistant writing out a prescription by hand, rather than using the computer-generated labeling system. To his surprise, Ost found that the assistant was writing the personalized label in Spanish, as she often did for the neighborhood's Hispanic population. Rather than berating her for circumventing standard operating procedures, Ost leveraged this knowledge into a change in routines by translating every computergenerated prescription into Spanish, thus opening up the market for a huge local clientele. Then, following a rapid influx of Vietnamese residents into the neighborhood, he added a third language to the computer program. These adaptive actions brought him even more recognition, and within a few years, Philadelphia Pharmacy was doing four times more business per square foot than the average American drugstore.

Glaveanu et al. (2013) note that artists often consider themselves "sponges," "90\% of the time in a receptive state", allowing themselves to be "impregnated" by things and people who thus enter an inner "factory of fermentation". The walks, voyages, readings they make and exhibitions they see all nourish creative impulses. This is exacerbated by the "tactile" nature they seem to possess, where "observation goes through the hand". Scriptwriters have habitually the attitude of a "hunter in the forest," always on the alert, always taking notes. Frequently, the starting point is connected to one's own history, the things seen as a child, the lived experience and the people one knows.

Horng and $\mathrm{Hu}(2008)$ note that culinary artists were able to inject the concept of fashion into their culinary designs. For example, furniture design, handicrafts, the style of kitchen utensils and home appliances, and the like often gave them new ideas. Little things such as soap dishes, tealeaf filters, and candle stands often stimulated them in unexpected ways. They also tended to be inspired by customers' likes and dislikes and their changing tastes, and by the menus of other restaurants. Moreover, they also felt that their reading and viewing 
of pictures and films, as well as their travel and the experience of other cultures afforded by it, were very important. They note an interview with a culinary artist: my work incorporates many sources of input: reading, art, life experience, culinary skills, famous cuisines, culture, and the like.

Horng and $\mathrm{Hu}(2008)$ mentions one interview with a culinary artist: "artists present their ideas through music or paintings, and we do it with our cuisine. We are more creative. I was writing to a girl and my tears dropped onto the letter. So, I made a teardrop-shaped choux pastry as a present for her. The ingredients included cheese, coco powder, sugar powder. I put all my affection into the product; I made the story of my love for her real. Like Dewey said, emotions increase the depth of design. That helps my work come to life."

Hargadon and Bechky (2006) note that, within the organizations they studied, individuals chose social interactions that retained the equivocality of past experiences - the multiple meanings that might be considered and reapplied in a new context. As one Hewlett-Packard engineer described: When you read about somebody's experience and then actually go and talk to them about it you find the level of knowledge is so much deeper than what can be transferred through a paper or an hour-long talk. There's a wealth of hidden knowledge that's a result of the struggles, the agonizing they went through to try to figure out what's the right way to proceed rather than the wrong way.

\subsection{Negotiation and Networking (Partnerships, Contacts, Relationships)}

Kerle (2010) notes leaders in organizations do not perceive creativity as something they do. Instead it is something they think about and coordinate. Creativity by its very nature requires action and context, is constructed around constraints and evolves out of practice. It is identified through perception, named creative by someone other than the person practicing it.

In studying craftsmen, Fillis (2007) points out that one type of craft owner/ manager is the businessoriented entrepreneur who is willing to take risks and recognises the importance of developing a customer base. Networking and relationship building are deemed very important for business success. This can be seen in the way in which the entrepreneurial owner/ manager makes an effort to visit overseas buyers in order to collect relevant export information. There is also a proactive tendency to develop skills and to monitor the environment for opportunities, as well as having the capacity to change direction, if needed, at short notice. Being part of a wider business and social network also assists in business development. In addition to providing potential sales, craft fair attendance can also serve as a way of collecting information about the market and new product development.

Sutton and Hargadon (1996) note how, whilst simultaneously being their client, Samsung and others partner with IDEO to develop their own innovation capabilities and culture. Fillis (2007) mentions one craftsman: "most of my stuff is export. I started off initially by weaving fabric, just weaving bolts of fabric and selling that to the designer labels in Italy and France. Basically, just knocking on doors. I started my first contact through the Princes Trust. I got a grant from the Princes Trust to go to Italy - a travel grant. They put me in touch with one or two people in the textile trade who said, right you should contact so and so. So it's basically by word of mouth. Meeting people who knew people who knew people." Lamacz and Gerchenzon (2012) note a case where persons in the wine industry were approached by a contact who asked them if they knew somebody from the wine industry able to create and to propose a wine list for a chain of luxury hotels. They accepted the offer by nominating themselves, and only subsequently established the capacity.

\section{Methodology}

To study the antecedents of opportunity, and the intricacies thereof, semi-structured interviews were conducted with 11 experienced entrepreneurs. The interviewees have been directly involved with innovation and entrepreneurship, with appropriate level of experience. The respondents were drawn from various industries. Although non-probability sampling procedures and purposive sampling were generally used, as many entrepreneurs as possible that meet the research requirements, were contacted to increase the sample's spread.

\section{Analysis}

Table 1 depicts the profiles of the respondents that emerged from the analysis. The respondents' profiles were based on the depth of discussion/ knowledge on a particular contributing factor, and the number of times a particular antecedent was referenced. As shown in figure 1, most of the respondents (6 out of 11) placed emphasis on the desire to solve problems as a key factor of opportunity recognition. Of equal importance to innovation were goals and aspirations (5 of the 11 respondents). Four of the respondents put emphasis on 
artistic creativity and adoption as antecedents of innovation. Networking, though not commonly seen as a key factor by some respondents in the identification of opportunities, was seen as an important precursor for innovation by 3 of the respondents. Interests, technology and first-person experiences ( 2 out of 11 each) and to a lesser extent, synthesis (1 out of 11), also aid in opportunity recognition.

Table 1. Respondents' profiles

\begin{tabular}{|c|c|}
\hline Respondent Number & Antecedent of opportunity \\
\hline 1 & Adoption \\
\hline 2 & Goals, interests \\
\hline 3 & Adoption, networking \\
\hline 4 & Problems, adoption, networking \\
\hline 5 & Artistic creativity, imagination, problems, goals, technology, adoption, networking \\
\hline 6 & Artistic creativity, problems, goals, synthesis, first person experiences \\
\hline 7 & Problems, goals \\
\hline 8 & Artistic creativity, interests \\
\hline 9 & Problems, goals \\
\hline 10 & Problems, technology \\
\hline 11 & Artistic creativity, problems, first person experiences \\
\hline
\end{tabular}

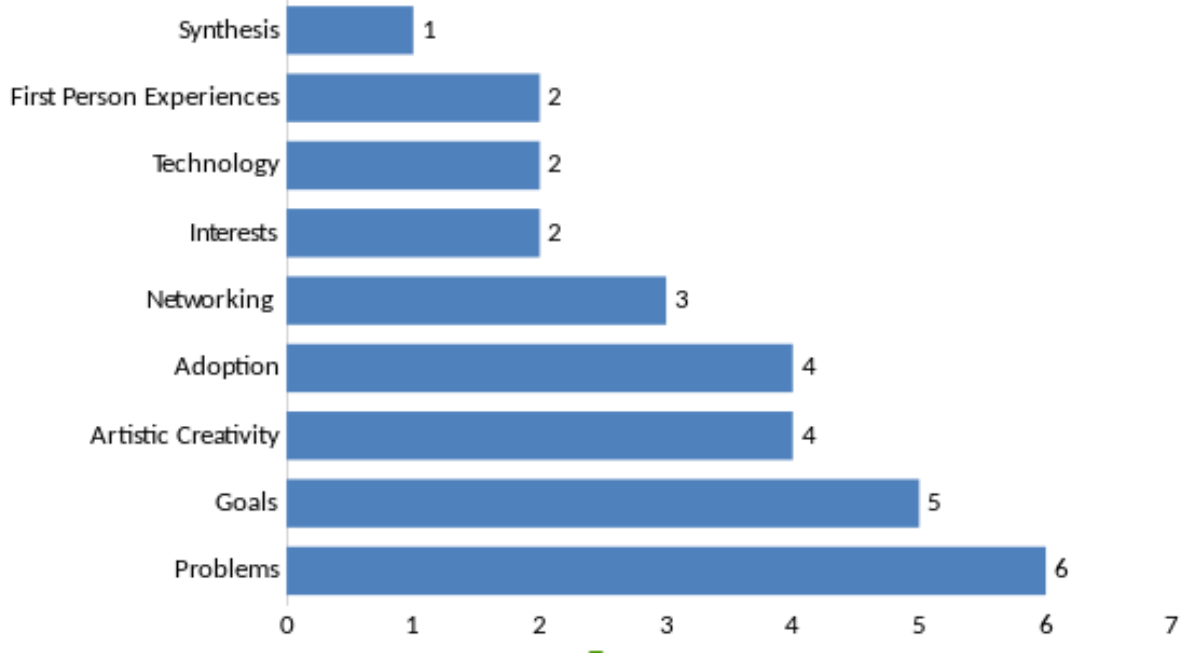

Figure 1. Relevance of the antecedents of opportunity

Below, each of the antecedents of opportunity is discussed in greater detail.

\subsection{Solving Problems - A Desire to Solve a Problem, or to Bring improvement or Change}

Solving problems, or a desire to bring improvement or change, can help with innovation. Being pressured by - being under pressure due to - a problem can inspire creativity. Discomfort and chaos can also create opportunities to think of ideas. Unhappiness about the status quo may aid with the recognition of opportunities. Desperation and hardships also drive innovation. It is taken that problems are generally accompanied by markets.

Some respondents insist on rather seeing problems as opportunities. The perspective that one decides to take on an issue or aspect is important. This can be positive or negative, and will impact on whether an individual sees an opportunity. Some may view the same problem to be without opportunity, whilst others see it as laden with potential. The innovator should maintain the right mindset. It is crucial to ask the right questions, to understand exactly what the problem is. The innovator may merely need to find a different solution based on a different perspective. Exposure to different cultures and contexts may help with this.

Complex problems or shunned problems, like poverty for example, may potentially provide vast opportunities for innovation. There may be a lot of opportunities in markets and areas generally overlooked by conventional business and players, for example under-resourced areas and poor neighbourhoods. Conventional wisdom would proclaim that these markets and areas do not contain a lot of opportunity.

Problems are very contextual. Simulating the problem may significantly contribute to solutions. Failure to conduct in-depth analysis may lead to a focus on the symptoms, rather than the cause of the problem. Proper research should be done to establish to what extent the problem has been interrogated before, what the 
results were, and what the current knowledge or thinking on the problem is. Certain problems may not have a simple solution. Furthermore, working on problems must be justifiable from a business perspective. People should not only focus on the problem that they want to resolve, but also consider the business implications for example, the innovation's relevance to the market as well as the size of the market. Outlets like newspapers may report problems that are everyday and thus very practical.

\subsection{Goals, Aspirations or Values}

Dreaming and aspirations can stimulate ideas. Problems people face may also lead them to start dreaming or to aspire. Values are seen to mostly contribute to opportunities that are sustainable. Values may thus indirectly result in dreams, visions or goals. One view is that the best kind of innovation occurs when there is a long-term perspective.

Goals may simply be tied to fads or trends - the objective would be to put down more sustainable goals. Goals may be set too ordinary. While the vision and goal need to be attainable, it is important to aim higher. Some feel goals should be unattainable, because they motivate and inspire. Thus, the more unrealistic a goal, the more it pulls the person up to higher levels. The general view is that more extraordinary and daring or challenging dreams and goals contribute more to innovation. On the other hand, a goal may simply be to do better than the status quo.

The person should also consider "silly", "insane" or "crazy", "childish" or "innocent" dreams and goals. The practical or possible should temporarily be discarded, conventional logic should be relaxed, and the impossible should not be excluded, when dreaming or setting goals. To devise goals, the mind should be emptied first. Goals require freedom to innovate - taking responsibility and allowing taking risks. Devising goals also require a proper understanding of the underlying, relevant concepts. Goals should be confirmed against the market.

The fact that goals are not always tangible, but abstract, may complicate buy-in and realization. It is not always easy to picture goals, and using goals may imply techniques or methods that not everyone may be comfortable with. Goals and visions may not initially enjoy support, and it may be necessary to build support first. Proper articulation of goals is crucial.

Taking the necessary steps to materialize dreams and goals is seen as important. A lot of perseverance is required to see dreams and goals through, and practical, incremental steps should be taken towards the dream or goal. The goal should constantly be to work towards a practical solution. In this regard, retrospection helps the process. Arrogance and a lack of knowledge may hinder the realization of dreams or goals. Given the inherent timelines that accompany goals, revisiting the goals on a time basis is important, in order to ensure that the innovation is consistent and realistic in alignment with where things currently stand in the environment, and still relevant and innovative.

\subsection{Artistic Ways, as Creative Inspiration, Imagination, or Objective Fantasy}

Most of the respondents agreed that artistic means can be used to innovate. They also shared the sentiment that creativity as artistic ways and imagination play a critical role in innovation and the generation of ideas. For one respondent, artistic ways is integral to innovation. Artistic creativity and imagination were also seen as offering the opportunity to differentiate and stand out.

Artistic creativity and imagination can also be applied in the use of current innovation, in different ways. It may not be necessary to try to find something that is absolutely new, and the best way to apply artistic creativity is to simply use and build on existing solutions to current problems that have already been identified.

“...to come up with solutions for current problems because there're a lot of problems around us these days and everybody is focusing on the new big thing that they can come up with and they are omitting all the opportunities of all the solutions they can find for problems right in front of them and everybody else." (Respondent 5).

Imagination is also seen as one of the big drivers when it comes to innovation. One respondent (Respondent 5) went as far as to say that people need to start using their imagination to a point where most people start thinking that they might be a bit childish and that the ideas they are considering won't work.

"I think to become more innovative people need to actually ... become like children, playing games like children used to play... If you look at children, they don't really have any inhibitions and as we grow up we create more and more inhibitions around the fact that what will society say of me if I say this or if I do that. So, we need to move away from that, also we need to be open to learn." (Respondent 5).

A dominant view is that with creativity, people need to completely step out of the norm of what they are used to doing, in order for them to become innovative. A lot of people are willing to operate - innovate and 
be creative - within the confines of their comfort zones, but are hardly willing to step outside of their comfort zones. Equally, stepping out of norms is crucial. In addition to the desire to step out, people need to have a better understanding of what it really means to step out of their comfort zones and norms. In some instances, this means not thinking and behaving according to the way one has been conditioned. In the business world, there may be the expectation to use (certain) business language and to behave in certain ways, and this may stifle innovation. People need to change their view of what is acceptable and aim to step out of their comfort zones and norms.

The innovator should play a lot, venture where he has not ventured before, and think on what he has not thought of before. The innovator needs to completely step out of what it is that he used to do or what he is used to doing. He should break with routines, and sustain the right attitude - remain positive. Continual and regular practice of creativity is seen as crucial. This involves looking for new problems to work on, and looking for new tools or approaches to work with. Also, it is important to ask the right questions.

Having a lot of references or examples greatly helps. Having different (global) references, whilst taking into account local cultural parameters, and being cognisant of the conditions in the market that is being focused on, are seen as key. Also, it pays to understand why something did or did not work in the referenced context. External references and learning from external resources can accelerate innovation. Similarly, switching perspective by adopting someone else's perspective, or a renowned perspective - in essence, using perspective templates - and doing so sequentially may also yield significant gains.

Getting a minimum viable product off the ground that the market can evaluate is seen as important. Requiring proper/ further research, the right contacts, and the right relationships, as well as difficulty or inertia to get out of comfort zones, may hinder the innovation effort.

Fear - of judgement, rejection, backlash, failure, the unknown or being uncomfortable - is also another dominant restraining factor. This limits the generation of ideas because a platform is not created where these "silly ideas" or "ideas that don't make sense" can be interrogated further. Thus, failure to step out of the comfortable zone, to move away from what is familiar and explore unfamiliar areas, can limit the generation of ideas. Failure itself can move the person forward - failure leads to learning and discoveries. Articulation and communication of creative intent are also important.

\subsection{Adoption - Borrowing from other Contexts}

The view is that industries frequently complement each other, by borrowing from each other. Adoption can lead to the identification of a solution that other people in the same industry are not looking for, and it will be unique. Adoption may accelerate innovation, in that it avails timely solutions. One benefit of using this approach is that people can benefit from the lessons learnt by the teams that have already made the innovation that is being adopted. This shortens the learning curve, which means that the person who uses this approach can move forward much quicker without having to repeat the whole process followed by the predecessors.

It is important to identify which problems have already been addressed in other contexts, so that it can be used as a base to determine the remaining gaps in the solution that should be targeted by innovation. It is important to understand the context of the innovation - the context a solution is adopted to.

"It's always good to understand the context or the market, what solutions have been addressed or what problems have been addressed in other countries, how the solutions worked, and it's sometimes the best idea not to re-invent the wheel, but to work with an existing partner that did it overseas and then work with them to innovate in a specific market. So that could be a good strategy." (Respondent 1).

Being open in thinking and to possibility, thinking holistically, and paying attention to context are seen as the crucial factors. Preconception hinders exploiting adoption. Being comfortable to move away from a focus on an industry that someone is familiar with and how things are done in that industry, as well as looking at completely disconnected industries, can aid in the identification of ideas and innovative solutions. The entails not looking at what other people in your industry or space are doing to find a solution. The solution that is being sought could be found in a totally different industry.

One view also is that, even if adoption does not directly lead to opportunities or solutions, it can do so indirectly. Simply by looking at other possible solutions, and solutions to other problems can inspire the innovator. Because it entails ideas and solutions that already work, and because a number of these ideas or solutions are browsed and perused, it may lead to the discovery of a better solution for a problem that the person is trying to solve.

"So, the benefits, if you look at a completely different industry to try and adopt an idea, you might find a solution for a problem that you weren't looking for that might even be a better or bigger benefit to you than what you were originally looking at." (Respondent 5). 
The target may very well be a product that has existed for a very long time, and that most people thus believe cannot be innovated anymore, and the source may be from a seemingly completely unrelated field seemingly not directly related to the product or problem at hand. Adoption may be about a solution's principle, rather than the solution itself. The reference may also be other things, like nature or natural phenomena.

"Someone that I think is really innovate as well is \$1 shave club. So, basically all \$1 shave club does is they sell you razor blades to shave with. They were bought by Gillett for 2 billion dollars. So how can a company selling razor blades that's been around for I don't know 20, 30, 50 or whatever years, all of a sudden come through and within 3 years or 5 years or however long they existed, but it was a short period of time, now all of a sudden just come and build a billion-dollar company using a product that's been in existence for years and years and years. So, that's not a new product, they just found a creative way to sell that particular product, but it was very innovate the way that they did it. So, you can't say that it's not innovate because they haven 't designed a product, the way the company was structured was innovative." (Respondent 2)

Simply copying others, but doing so in an innovative way, may also be seen as innovation. Innovation does not necessarily imply that you have to be number one. The frame of reference is seen as important.

"So, you didn't have to invent the cell phone, but Samsung copied, Blackberry copied, Apple copied, everybody copied Nokia." (Respondent 3 )

A benefit of adoption is that the innovator starts with something that already works. Communication across borders, like industry borders, breaks down boundaries, and thus accelerates the process. It is necessary that the innovator leaves his regular environments and common routines.

A potential disadvantage may be that it may take a significant amount of search, and thus time, to discover new solutions. Discovering a solution also is not guaranteed. The context from which an idea is being adopted forms a frame of reference, and it is important to understand what the benefits of the concept you are trying to adopt are, as well as understanding how it operates, before it can be adopted and adapted in a different context.

Another challenge is failure to adapt what is being borrowed from another context into the new environment. Overall, the entire process entails more than just adopting the basic principles. Thus, the risk is that, without adequate adaptation, the solution may not work as well as expected in the new environment.

"One of the difficulties of doing this is that you don't adapt. You know it's far more than adoption. You adopt the basic principle and then you have to adapt it for the new environment that you're bringing it into. So, for example, I've adopted manufacturing factory techniques in an intensive care unit, but I've obviously had to adapt them for that new environment. So now I think people sometimes forget to adapt." (Respondent 4).

\subsection{Negotiation and Networking}

Some respondents see networking as the most important tool of life, and the most valuable tool of innovation. Networking can multiply the innovation effort, particularly if certain levels of trust and companionship have been reached and established. A group may innovate more effectively than an individual, and a group that connect well with each other may innovate even more effectively.

"That's the most important thing that you have of in all of the tools you have in life. Being able to connect with people is the most valuable tool to have innovation. Remember for one person to be innovative is ok. If you put 3 or 4 people in a room and you say to them, just you now start creating, it will also be good. But if you can get those 3 or 4 people in the room to first connect with each other, to actually start trusting each other, to feel comfortable and to know that there's no judgement in that room, you will find that creativity explodes because then they don't mind if they say something stupid or say something crazy." (Respondent 5).

Trust, coupled with freedom of expression, are seen as important contributors to the success of networking. Networking, which is based on trust for one another, paves the way for innovation, because people can open up and share any idea that they have without fear that they may say something that is deemed silly. People are less inhibited from saying what they want to say. Networking is seen as not simply connecting with people. Rather, it involves understanding who the people are you are meeting with, what their backgrounds are, as well as whether you can relate to them on the basis of values. Networking is based on quality and authentic relationships, like-mindedness, resource sharing, and sharing of values, and seeks mutual benefit and support.

Networking can enable people to become successful. Networks can introduce someone to resources otherwise unavailable, and can shape his mindset. Being put in contact with people who have experience - in a particular industry, for instance - as well as being mentored, can accelerate the innovator and innovation 
process. Increasing the number of people working on a problem may increase perspective, and thus the number of solutions and time to solutions - simply put, 2 heads are better than 1 . Also, people can use networks to learn and gain knowledge, discover new opportunities, and benefit from more successful and experienced people.

Networks may very well suggest opportunities. Conversations can lead to a lot of innovative ideas. Through networking people may discuss problems or ideas that they find interesting, without necessarily thinking of them as opportunities; conversely, the innovator finds it very opportune. Also, others may suggest solutions and ideas to the innovator whilst discussing his problems, without even realizing it.

Still, the efficiency and quality of the network should be managed. And there must still be problem solving and creativity skills embedded in the network. The issue with networking or negotiation is that, even though he may do so once or twice, the innovator cannot constantly rely on the outside for opportunities. The innovator will have to develop innovative competencies internally at some point.

Networking demands relationship building - investing in relationships eventually boosts innovation. It may take time to build networks based on trust. Networking also requires humility - the innovator must be teachable or coachable, and an apt listener. The innovator must still evaluate the views and opinions of others, and must have the ability to do so. It is vital to connect with the right people - people that will actually contribute to the innovator's experience. The number of connections the innovator has is not necessarily the most important factor. Talkers are distinguished from doers. Networks can be draining as well. The innovator may find that the type of network he requires is non-existent, and may need to build his own network evangelize - first. He may have to first raise awareness and get commitment.

\subsection{Interests, Inquisitive Journeys or Passions}

Interests enable people to focus on their strengths and they will be more successful if they have an interest, or are inquisitive and passionate about something. Focusing on something of interest helps with motivation. Hobbies are also seen as paths to innovation. Some respondents encourage people to have as many hobbies, or try as many experiences as possible, because it is known to lead to creativity and innovation.

An inquiring mind is equally seen as critical to the generation of ideas. Having an inquiring mind may also mean to have a mind that is looking for opportunity all the time. Sometimes an inquiring mind entails collecting information, without having a real sense of how it can be used, but over time that piece of information can trigger an eureka moment as things fall into place, and that information suddenly becomes invaluable.

Passion is cited as one of the main factors that can drive innovation, because it is driven by an inner energy which propels people to fulfil their goals or aspirations. Necessity may also drive motivation, and can thus stimulate business ideas. Passionate people are seen as doers and implementers. With innovation, the outcome is determined by the amount of energy you put, and passion contributes to this.

At the same time, passion is critiqued in that passion may not be accompanied by (business) intelligence and common sense. Being driven by passion alone can be dangerous and may lead nowhere. Being overly passionate about something may also cause the person to become ignorant. The big picture should constantly be kept in mind.

\subsection{First Person Experiences}

First person experiences were seen as an important aid in identifying ideas. Some respondents saw their first-person experiences as "brilliant for defining solutions". One respondent established a first business through first person experiences. Life experiences, like upbringing, circumstances faced, or events, may also contribute to ideas.

First person experiences may point to experiences shared by others, and this can point to viable opportunities. Having experienced, and experiencing something first hand may give the innovator an edge during the innovation and marketing process. The offering may be seen as more authentic and genuine, and the innovator may constitute proof of concept. It becomes easier for the innovator to identify with people experiencing a similar problem, and in turn, customers may more easily identify with the person. Very personal experiences may also be strong sources of motivation and drive to innovate. Negative first-person experiences may lead to necessity, and often motivate people to do something about their situation, thus leading to innovation.

“... her daughter had cerebral palsy and out of that she's established the most amazing business, designing, and distributing wheelchairs. A whole industry around mobility, you know. So, there's no doubt that, you know, if you'd have said to [Person name] 5 years before her daughter was born with cerebral palsy and therefore would need a wheelchair, that she was going to be in that 
business, she would've have said no... So, it's very much a first-person experience, necessity is the mother of invention" (Respondent 4).

"Global" experiences - experiences at higher levels, like the corporate or macro-level, likely shared by a number of people, may also contribute to innovation. This points to the spirit or ethos of a group or society at a particular point in time. The example of war, and innovation that usually stems out of war was raised. Competition - competing with others - may also constitute and become a first-person experience that fuels innovation.

First person experiences may be unique and opportune. First person experiences are more powerful when accompanied by learning. Difficult, painful or intense experiences can significantly aid learning. Personal experience can also contain perception and learning otherwise not possible. First person experiences may lead to personal or tacit understanding of a situation or phenomenon. The knowledge and level of understanding that comes with a personal experience cannot be transferred to other people even if it's written down, but provides the best lesson for the person who is affected. The more one focuses on experiences, the closer one gets to phenomena. Personal interpretation of phenomena is seen as important. Diverse sources of experiences and going out of your way to have experiences, can aid first person experiences. For example, window shopping is also seen as a way to increase experiences.

On the other hand, the personal experience may not be a sufficiently shared experience, such that it may be marketed. Even when the experience launches the person into action, the person may fail to truly innovate, if he simply reverts to current solutions. Similar to passion, and the drawbacks of passion, the person may become too engrossed in his own experience. The experience may cloud judgement. Again, business intelligence and acumen are crucial. It is still necessary to be open and be willing to consider others' views and opinions.

\subsection{Scientific or Technical Inquiry or Development}

The view is that technology in itself allows innovative solutions. It may not be necessary to develop (radically) new technology per se. Technology can be borrowed and studied, in order to repackage it and apply it in new ways to existing problems.

Innovation can have a multiplicative effect - in general, innovation may lead to more innovation. The technology embedded in new innovation and communities can stimulate new ideas. Studying what other people have already done or attempted can accelerate the process, and may also be a source of ideas. It is also possible to consider, implement and learn from technology used by other industries. Studying how people are using technology is very rewarding.

Science and technology-based innovation require new science and technology to build on. Science and technology-based innovation also require a base of scientists, technical workers and engineers, etc., who have the required knowledge to innovate in this way. Subject knowledge or technical knowledge is required to innovate in individual fields. Science and technology increases the ceiling of what can be achieved through innovation. Science and technology avail experts that the innovator can draw from.

Looking to technology leaders, coaching and mentoring, further develops process, skills and capability specific to technology innovation. Access to technology super-specialists is a significant bonus - it may accelerate further learning and innovation. Being coached by those who are already successful, and working with people who are smarter than you, are important factors, as it opens up unique perspective.

Both a narrow focus and a too broad focus may hinder technology-based innovation. It may quickly become unfocused, given all the knowledge available. At the same time, only narrowly focusing on and following technology may deliver insignificant results. A related challenge is getting stuck in the process of research and analysis (analysis paralysis), to an extent that the person fails to take the next step and produce something. It is easy to think too technically.

Science and technology must still be reworked into products. To overcome this, the aim should be to develop a minimal viable product (MVP) as soon as possible, so that customers can test the product and provide feedback that can be used to further design and develop the product. Involving the customer as early as possible, and developing solutions with the customer, help. Creativity may equally be realized customer-side, by incorporating customers and their feedback.

\subsection{Synthesis and Induction}

Because synthesis brings concepts, ideas, processes or products together, it may result in coming up with new solutions relatively quickly. A benefit of synthesis is that the innovator can quickly learn from others. Synthesis may result in completely different products. Synthesizing existing products may form richer composite products. Synthesis can also be used to revive obsolete products. For instance, the components of a 
product can be re-configured, to form an altogether new product.

Synthesis is also a welcome solution when faced with abrupt changes in product or industry. Synthesis can also be used to create new concepts and knowledge that in turn can be used to innovate from. When done right, synthesis should lead to the sum being greater than the parts.

It is also seen that synthesis requires taking a step back and looking at a product or concept, for instance, not just for what it is, but what else it can be paired with to develop something novel. That is, delineating a product's fundamental properties, and finding other products that it can be combined with.

"A lot of people will say "well what can you do with a phone? Well it's only a phone." And if you look at it as it's only a phone, then you're not going to find innovation. But if you look at it as an electronic device that you carry around and you say well what other electronic devices do we have? ... well I've got a voice recorder, I've got a phone, I've got a camera, I've got a video recorder, I've got a TV remote and you start looking at all the different electronic things and you say well "how now can you make that all one?" (Respondent 5 )

A simple method is to simply try to combine different things and see if they will fit together. Another technique is to seek, note and cross-reference best cases, to apply them to the current situation. This involves finding out how the concept was applied initially, how the concept was received by the market, as well as how well it performed. Also, focusing on a weakness in a concept, idea, process or product, and looking to synthesis to negate these weaknesses, or even turn them into new strengths, can lead to new innovative solutions. Market intelligence and acumen may also guide the synthesis process - serve as a source of suggestions as to what can be successfully combined. It is crucial to take the customers' perspective into consideration.

Synthesis increases the complexity of the new product. The loss of simplicity may impact on the resources, time, focus and costs associated with getting the product to market. There is also the danger of over-synthesising the features, so that the product may become too complicated for the user. It is possible to lose sight of the market.

Attempts may also be made to combine things that generally cannot be combined - not everything can be combined. Synthesis may lead to the creation of inefficient products or processes, and there may be a tradeoff between specialisation and cost. Synthesis may also hinder a specialization focus. Proper integration and adaption are required to ensure the solution is scalable. Attention should be paid to the market, and local market characteristics. For example, the combination of green chilli (sushi paste) and ice cream works very well, but mostly in Japan.

"This was a more regional thing, in Japan ice cream, with you know that green chilli that you use on sushi, what's it called?... So, there they combined it... Yeah, it's disgusting but it works regionally. So, you took something from sushi and ice-cream and you put them together. It works." (Respondent 3)

\section{Conclusion}

The desire to solve problems was seen as a leading factor in the generation of ideas and recognition of opportunities by most of the respondents. Goals and aspirations also play a key role in opportunity recognition and innovation. Problems can form the basis for goals and aspirations to come up with a solution or an improvement. Emphasis was also placed on artistic creativity and adoption as antecedents of idea generation and innovation. Other contributing factors, such as networking, interests, technology, first-person experiences and synthesis also play some role in opportunity recognition and innovation.

Each of the antecedents potentially offer and allow expert areas of innovation. The innovator may become very experienced in a particular antecedent as an area of innovation. The innovator may continually gravitate towards or focus on a limited number of antecedents - one or two antecedents - to identify opportunities, and to innovate. Being more experienced in a particular antecedent should suggest that the innovator is able to better exploit the antecedent than other innovators.

A number of the antecedents may offer unique solutions and benefits. Also, a number of the antecedents are noted to accelerate innovation. In certain contexts, one antecedent may be more applicable or beneficial than another. However, none of the antecedents are sufficient for innovation - none of the antecedents by themselves can necessarily ensure innovation. Each of the antecedents has caveats that restrain their ability to impact innovation. For instance, passion is important for innovation, but blind passion may equally be dangerous.

Themes such as business intelligence and acumen, as well as "silly ideas", "crazy ideas", "thinking, behaving or playing like a child", and the mention of comfort zones and norms, are common to the antecedents, and suggest that there may be a number of principal factors underlying the antecedents studied. Underlying all 
the antecedents may be the need to cross boundaries of the acceptable, or rather boundaries of the possible.

A relationship should exist between what is seen as acceptable (the acceptable), and what is seen as possible (the possible). In many ways, crossing the line of what is seen as possible constitutes crossing the line of what is seen as acceptable, and this is both the obstacle and objective of the innovator. Hence, innovation can equally be defined as crossing the boundaries of what is possible, whilst still yielding outcomes that are desirable. Boundaries of the possible - what defines, determines, and constraints what is possible - may not be obvious, and may require a combination of a unique perspective, scholastic enquiry, and empirical experience to transcend. This is also something that deserves further investigation.

The primary factors expected to underlie the antecedents are: 1) perspective, 2) knowledge, and 3) first person experience (sensing, perceiving, interpreting, comprehending). Perspective helps to change the worldview, to permit a new frame of reference. Still, knowledge in the form of axioms, laws and methods are necessary. Even if the perspective is changed, and even if a change in perspective can realize innovation, it is accomplished through knowledge - knowledge still remains a medium. Furthermore, the innovator may only be able to really grasp the phenomenon he wishes to innovate, after personally experiencing it and interacting with it - at this point additional knowledge is created and added. Personal experience, experiences, and experiencing help to consider all the input, data and information. There may be certain forms of knowledge vital for innovation that are only obtainable through first person experiences.

Not all respondents truly actively differentiate between the factors. Only when discussing them do they note the differences. There may be a link between the expertise of the innovator, and the number of antecedents considered by the innovator. It seems that more experienced entrepreneurs generally span the antecedents to a greater extent. There may be a correlation between ease of use of antecedents or antecedent experience, and actual entrepreneurial experience.

Also, diversity in the entrepreneur's scope may play a role. Some entrepreneurs are particularly focused on specific markets. At the same time, the nature of innovation is not expected to be dependent on the market segment. Antecedents considered by innovators may shape and define the type and nature of innovation taking place in a sovereign.

\subsection{Recommendations for Future Research}

Future research can examine whether such antecedent-based innovation specialization indeed exists, the extent by which innovators specialize in their innovation skills, and the extent by which innovators stick to their type, style, or specialization of innovation. Although perhaps more ambitious, future research can also examine whether there exists certain specific innovation orientations or inclinations within countries.

In addition, future research can look at formal definitions of the antecedents, and perhaps extending or changing the antecedents. Also, it can examine how parsimonious the antecedents are, as well as the structure and inter-relationships of the antecedents of opportunity. Of course, future research can further investigate boundaries of the possible.

\section{References}

Ardichvili, A., Cardozo, R. and Ray, S., 2003. A theory of entrepreneurial opportunity identification and development. Journal of Business venturing, 18(1), pp.105-123.

Baron, R.A., 2007. Behavioral and cognitive factors in entrepreneurship: Entrepreneurs as the active element in new venture creation. Strategic Entrepreneurship Journal, 1(1-2), pp.167-182.

Chodorow, J., 2006. Active imagination. The handbook of Jungian psychology: Theory, practice and applications, pp.215-243.

DeTienne, D.R. and Chandler, G.N., 2004. Opportunity identification and its role in the entrepreneurial classroom: A pedagogical approach and empirical test. Academy of management learning \& education, 3(3), pp.242-257.

DiLiello, T.C. and Houghton, J.D., 2006. Maximizing organizational leadership capacity for the future: Toward a model of self-leadership, innovation and creativity. Journal of managerial psychology, 21(4), pp.319-337.

Fillis, I. and Rentschler, R., 2010. The role of creativity in entrepreneurship. Journal of Enterprising Culture, 18(01), pp.49-81.

Fillis, I., 2007. An examination of Celtic craft and the creative consciousness as a contribution to marketing creativity. Journal of Strategic Marketing, 15(1), pp.7-16.

Gentner, D., 2010. Bootstrapping the mind: Analogical processes and symbol systems. Cognitive Science, 34(5), pp.752-775. 
George, J.M., 2007. 9 Creativity in Organizations. The academy of management annals, 1(1), pp.439-477.

Glaveanu, V., Lubart, T., Bonnardel, N., Botella, M., de Biaisi, P.M., Desainte-Catherine, M., Georgsdottir, A., Guillou, K., Kurtag, G., Mouchiroud, C. and Storme, M., 2013. Creativity as action: findings from five creative domains. Frontiers in psychology, 4.

Grant, A.M. and Berry, J.W., 2011. The necessity of others is the mother of invention: Intrinsic and prosocial motivations, perspective taking, and creativity. Academy of management journal, 54(1), pp.73-96.

Hargadon, A.B. and Bechky, B.A., 2006. When collections of creatives become creative collectives: A field study of problem solving at work. Organization Science, 17(4), pp.484-500.

Hirst, G., Van Knippenberg, D. and Zhou, J., 2009. A cross-level perspective on employee creativity: Goal orientation, team learning behavior, and individual creativity. Academy of management journal, 52(2), pp.280-293.

Holyoak, K.J. and Thagard, P., 1997. The analogical mind. American psychologist, 52(1), p.35.

Horng, J.S. and Hu, M.L., 2008. The mystery in the kitchen: Culinary creativity. Creativity Research Journal, 20(2), pp.221-230.

Kerle, R. 2010, Creativity in Organizations. The Creative Leadership Forum [online] Available at: http://static1.1.sqspcdn.com/static/f/110188/9602486/1291014610303/Creativity+in+Organisations+ MarkedUp.pdf?token=26oSGqsfeQECra2muQ98xbIOw6c\%3D [Accessed on 10 March 2018].

Kerr, C. and Lloyd, C., 2008. Pedagogical learnings for management education: Developing creativity and innovation. Journal of Management \& Organization, 14(5), pp.486-503.

Kozbelt, A., 2008. Hierarchical linear modeling of creative artists' problem solving behaviors. The Journal of Creative Behavior, 42(3), pp.181-200.

Lamacz, J. and Gerchenzon, G., 2012. Business creation: from theory to practice: Case study on a wine merchant company creation process.

Lumpkin, G.T. and Lichtenstein, B.B., 2005. The role of organizational learning in the opportunity-recognition process. Entrepreneurship theory and practice, 29(4), pp.451-472.

McFadzean, E., 1998a. The creativity continuum: Towards a classification of creative problem solving techniques. Creativity and Innovation Management, 7(3), pp.131-139.

McFadzean, E., 1998b. Enhancing creative thinking within organisations. Management Decision, 36(5), pp.309-315.

Moran, S., John-Steiner, V. and Sawyer, R., 2003. Creativity in the making. Creativity and development, pp.6190.

Rank, J. and Frese, M., 2008. The impact of emotions, moods, and other affect-related variables on creativity, innovation and initiative in organizations. Research Companion to Emotion in Organizations, (New Horizons in Management Series).

Richards, R., Kinney, D.K., Benet, M. and Merzel, A.P., 1988. Assessing everyday creativity: Characteristics of the Lifetime Creativity Scales and validation with three large samples. Journal of Personality and Social Psychology, 54(3), p.476.

Sawyer, R.K., 2000. Improvisation and the creative process: Dewey, Collingwood, and the aesthetics of spontaneity. The journal of aesthetics and art criticism, 58(2), pp.149-161.

Sternberg, R.J., Kaufman, J.C. and Pretz, J.E., 2001. The propulsion model of creative contributions applied to the arts and letters. The Journal of Creative Behavior, 35(2), pp.75-101.

Sternberg, R.J., Kaufman, J.C. and Pretz, J.E., 2003. A propulsion model of creative leadership. The Leadership Quarterly, 14(4), pp.455-473.

Sutton, R. I., 2002. Weird ideas that work. New York: The Free Press.

Sutton, R.I. and Hargadon, A., 1996. Brainstorming groups in context: Effectiveness in a product design firm. Administrative Science Quarterly, pp.685-718.

Tschang, F.T., 2003. When does an idea become an innovation? The role of individual and group creativity in videogame design. DRUID Summer Conference, Copenhagen, 12-14 June 2003, pp.1-26. Research Collection Lee Kong Chian School Of Business.

Vise, D., 2007. The google story. Strategic Direction, 23(10).

Ward, T.B., 2004. Cognition, creativity, and entrepreneurship. Journal of business venturing, 19(2), pp.173188.

Yokochi, S. and Okada, T., 2005. Creative cognitive process of art making: A field study of a traditional Chinese ink painter. Creativity Research Journal, 17(2-3), pp.241-255.

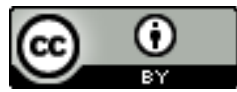

